\title{
RED REGIONAL DE TECNOLOGÍA EN ARQUITECTURA
}

La Red Regional de Tecnología en Arquitectura se creó en el año 2006, con la asociación de cinco Facultades de Arquitectura, tres de Argentina (Universidades Nacionales de La Plata, del Litoral y del Nordeste), una de la Universidad Nacional de Asunción del Paraguay y una de la Universidad de la República del Uruguay. La Dirección de la Red está a cargo del Arq. Jorge Lombardi, de la Facultad de Arquitectura y Urbanismo de la Universidad Nacional de La Plata. El objetivo principal de la Red es la difusión del trabajo cotidiano, de docencia, investigación y extensión, del Área de la Tecnología en estas Facultades y en todas aquéllas cuyos docentes quieran adherir a la Red Regional, en razón de que las adhesiones son personales y no institucionales.

Como una manera de promover la difusión de estos trabajos se han realizado hasta la fecha seis Congresos Regionales de Tecnología en Arquitectura, cuya séptima edición tiene por sede la Facultad de Arquitectura y Urbanismo de la Universidad Nacional del Nordeste.

La Red Regional trabajó arduamente para poner en marcha una Maestría en Tecnología de la Arquitectura. Lamentablemente, la propuesta no tuvo aceptación por parte de la CONEAU, por lo que la Facultad de Arquitectura, Diseño y Arte de la Universidad Nacional de Asunción del Paraguay tomó la iniciativa de promover su puesta en marcha en su sede, y es así que desde marzo del año pasado (2014) se está dictando normalmente en dicha Casa de Estudios. En sector aparte de este número se exponen las características de esta Maestría.

Adherirse a la Red Regional de Tecnología en Arquitectura no agrega nada al currículo de cada uno, pero contribuye a difundir nuestro trabajo, estar presente en todas estas manifestaciones, y trabajar mancomunadamente para perfeccionar nuestras metodologías de enseñanza y de aprendizaje, optimizar la labor de investigación y difundir los resultados en actividades de extensión. 